AperTO - Archivio Istituzionale Open Access dell'Università di Torino

\title{
Ab initio simulations and experimental Raman spectra of Mg2SiO4 forsterite to simulate Mars surface environmental conditions
}

This is a pre print version of the following article:

Original Citation:

Availability:

This version is available http://hdl.handle.net/2318/1669146

since 2018-05-30T16:16:05Z

Published version:

DOI:10.1002/jrs.5127

Terms of use:

Open Access

Anyone can freely access the full text of works made available as "Open Access". Works made available under a Creative Commons license can be used according to the terms and conditions of said license. Use of all other works requires consent of the right holder (author or publisher) if not exempted from copyright protection by the applicable law. 


\title{
$A b$ intio simulations and experimental Raman Spectra of $\mathrm{Mg}_{2} \mathrm{SiO}_{4}$ forsterite to simulate Mars surface environmental conditions
}

\author{
C. Stangarone ${ }^{1}$, U. Böttger ${ }^{2}$, D. Bersani ${ }^{1}$, M. Tribaudino ${ }^{1}$, M. Prencipe ${ }^{3}$, \\ ${ }^{1}$ Physics and Earth Science Department, University of Parma, Parma, Italy \\ ${ }^{2}$ Institute for Planetary Research, DLR, Berlin, Germany \\ ${ }^{3}$ Earth Science Department, University of Turin, Torino, Italy
}

Corresponding email author: claudia.stangarone@studenti.unipr.it

Keywords: forsterite, ab initio modelling, Raman spectroscopy, temperature, ExoMars

\begin{abstract}
In this study present full Raman vibrational pattern of $\mathrm{Mg}_{2} \mathrm{SiO}_{4}$ forsterite, computed from first principles, employing the hybrid Hamiltonian WC1LYP by means of CRYSTAL14 code at static equilibrium and at $0 \mathrm{~K}, 300 \mathrm{~K}$ and $1000 \mathrm{~K}$. The simulations are compared to the available literature data, confirming the accuracy of the calculations, and to experimental Raman spectra taken at room temperature on a natural sample of forsterite $\left(\mathrm{Mg}_{1.76} \mathrm{Fe}_{0.22} \mathrm{SiO}_{4}\right)$, in order to verify if this method is suitable also for compositions slightly different from endmembers.

The comparison with the experimental data at room temperature shows a very good agreement (an average discrepancy of $7 \mathrm{~cm}^{-1}$ ) and it allows correct symmetry assignment of Raman features to specific vibrational modes. A quantum mechanical mass substitution of calculated data is performed for each of the six symmetry-independent cations within the unit cell $\left({ }^{56} \mathrm{Fe}\right.$ for ${ }^{24} \mathrm{Mg}$ within the M2 and $\mathrm{M} 1$ sites, ${ }^{30} \mathrm{Si}$ for ${ }^{28} \mathrm{Si}$, and ${ }^{18} \mathrm{O}$ for three ${ }^{16} \mathrm{O}$ within the tetrahedron) in order to study the contributions of each elements to the overall vibration. The aim is to relate the major experimental peaks, not only to specific structural features but also to link them to the occurring Raman shifts due to temperature and composition.

Moreover, in order to foresee the possible response of Raman spectra to the different environmental conditions occurring on planetary surfaces, i.e. Mars, we calculate full Raman spectra at 0K, $300 \mathrm{~K}$ and $1000 \mathrm{~K}$ including zero point effects. These results may steady a Raman database and provide new clues to improve the interpretation of data acquisitions during the 2018 ExoMars mission, which will carry on board a Raman Laser Spectrometer (RLS).
\end{abstract}


Introduction

The upcoming space missions to Mars stems from the development of new technological instruments and methodology capable of in-situ surface explorations. On this view, Raman spectroscopy is by now an established technique for extracting mineralogical and compositional information, without requiring any preparation of the sample or vacuum, in order to perform a nondestructive in-situ analysis. The second ExoMars European-Russian space mission, scheduled for 2018, will carry on board of the Rover unit a Raman Laser Spectrometer (RLS). The current study aims to improve the Raman database and steady a theoretical background on some issues that are still unresolved from simple laboratory experiments, employing ab initio computational methods.

The object of this study is forsterite, an end member and major component of the olivine group. Olivine is a widespread mineral in Martian meteorites (Wang) and thus, by inference, on the surface of Mars. It is an orthosilicate, with general formula [M(1) $\mathrm{M}(2) \mathrm{SiO} 4]$, where both $\mathrm{M}$ sites are occupied by $\mathrm{Mg}, \mathrm{Fe}^{2+}$ and $\mathrm{Mn}^{2+}$ for most natural crystals. Most rock-forming olivines are binary $\mathrm{Fe}-\mathrm{Mg}$ solid solutions consisting of the isostructural endmembers forsterite (Fo), $\mathrm{Mg}_{2} \mathrm{SiO}_{4}$, and fayalite $(\mathrm{Fa}), \mathrm{Fe}_{2} \mathrm{SiO}_{4}$.

Forsterite is also an important rock-forming silicate and is the most abundant phase of Earth's upper mantle. Therefore, due to its petrological and geophysical significance, it has been the subject of several other studies, most of them focused on its structure, crystal chemistry and thermodynamic; for examples investigations at high-temperature and high-pressure or inelastic neutron scattering studies (Rao et al. 1988; Ghose et al. 1991; Price et al. 1992; Schmidt et al. 1992). Among these studies, vibrational spectroscopy and lattice dynamics were extensively employed on forsterite, because its small unit cell and relatively simple orthosilicate structure afford detailed analysis (citare tutti I lavori). However, due to the low intensity of the modes below $600 \mathrm{~cm}-1$ a correct mode assignment was hardly done, also for the mixed nature of the modes; besides in the diagnostic Si-O stretching modes at higher wavenumber, the contribution of the different oxygen in the tetrahedron for each mode were not assessed. the Hohler and Funk (1973) observed, for example, strong mode mixing at low wavenumbers, and they considered the nature of crystal-field and dynamical splitting of the internal $\mathrm{SiO}_{4}$ vibrations. Stidham et al. (1976) measured the single-crystal Raman and IR spectra of synthetic end-member tephroite at 300 and $14 \mathrm{~K}$ and confirmed earlier proposals that internal $\mathrm{SiO}_{4}$ modes do not interact greatly with the low-energy M-site cation vibrations. Piriou and McMillan (1983) made Raman measurements on different polycrystalline olivines and related phases. They focused their analysis on the high-wavenumber region and discussed the nature of mode coupling between internal $\mathrm{SiO}_{4}$ vibrations as well. 
Few years later Chopelas (1991) undertook a single-crystal Raman study of natural and synthetic olivines, including forsterite, fayalite, and monticellite, emphasizing the low-wavenumber modes below $450 \mathrm{~cm}^{-1}$, and she presented new vibrational mode assignments. New hints for a correct mode assignment arrived in 1973 when $\mathrm{Pa}^{\wedge}$ ques-Ledent and Tarte made some of the first powder IR and Raman measurements on synthetic endmember forsterite using ${ }^{28} \mathrm{Si}^{30}{ }^{30} \mathrm{Si}$ and ${ }^{24} \mathrm{Mg}-{ }^{26} \mathrm{Mg}$ isotopic substitution in order to assign the observed bands. However, they did not present their Raman spectra. More recently, Kolesov and Geiger performed in 2004 another experimental isotopic substitutions for $\mathrm{Mg}$ presenting spectra and new mode assignment, in order to clarify $\mathrm{Fe}-\mathrm{Mg}$ partitioning within M1-M2 sites.

On the other hand, several studies on the effects of temperature, pressure and composition by means of vibrational spectroscopy have been done in order to link the Raman peaks to the structure. Chopelas (1990) carried out some of the earliest Raman measurements at high pressures in a diamond anvil cell to about $20 \mathrm{GPa}$ on forsterite in order to investigate its thermodynamic properties. Liu and Mernagh (1993) recorded high-pressure Raman spectra to $17 \mathrm{GPa}$ on fayalite and $30 \mathrm{GPa}$ on forsterite. Gillet et al. (1991) undertook a high-temperature Raman study on forsterite to about $1150 \mathrm{~K}$ and $10 \mathrm{GPa}$ and further in 1997 made further high-temperature measurements on forsterite up to $2000 \mathrm{~K}$ in order to investigate the intrinsic anharmonicity of the Raman-active modes. Several computational studies AGGIUNGERE: [A previous ab initio study has been already done by Noel 2006 but no intensities and an incorrect symmetry assignment and comparison with experimental data, plus a generically mode attribution not focused on Raman activities].

The aim of the current study is to perform a correct analysis and attributions of the vibrational pattern especially for those modes at low wavenumbers and a physically consistent forecast of the Raman shift responses to increasing temperature. By means of the latest realisation of CRYSTAL code, it is possible to simulate a full Raman pattern (frequencies and relative intensities). The calculated spectrum for forsterite was compared to the spectra calculated after isotopic substitution for each symmetry-independent cations in the unit cell $\left({ }^{56} \mathrm{Fe}\right.$ for ${ }^{24} \mathrm{Mg}$ within the M2 and M1 sites, $\mathrm{Si}$ for $\mathrm{Si}$, and three ${ }^{18} \mathrm{O}$ for ${ }^{16} \mathrm{O}$ for tetrahedron). Doing so, symmetry assignments in literature can be corrected also at low wavenumbers, where the low intensities peaks and the coupled mixed modes hinder a correct attribution. Moreover, the high temperature spectrum of forsterite at $1000 \mathrm{~K}$ was calculated, and compared to the high temperature investigation on a natural sample of olivine, with a composition of $\mathrm{Mg}_{1.76} \mathrm{Fe}_{0.22} \mathrm{SiO}_{4}\left(\mathrm{Fo}_{\# 89}\right)$. The good agreement with experimental and literature data allowed us to start calculations including zero point effects introducing in our 
calculation temperature, in order to simulate planetary environmental conditions. It also provides valuable insight that can be used to interpret or understand the macroscopic thermodynamic functions like heat capacity and bulk physical properties such as thermal expansion and compressibility.

\section{Experimental}

Computational details

Geometry optimization and spectral calculations on forsterite are performed by using the CRYSTAL14 program (Dovesi et al. 2014), which employs localized basis sets. Computations are made at the hybrid Hartree-Fock/density functional theory (DFT) level, employing the WC1LYP (Wu \& Cohen 2006, Lee et al. 1988) exchange-correlation functional, which includes a percentage (16\%) of the exact non-local Hartree-Fock electron exchange. The Hartree-Fock exchange is added in order to correct for the self-interaction error (the interaction of an electron with itself), which is typical of both purely DFT local density (LDA) or generalized gradient approximation (GGA) functionals. The use of hybrid Hartree-Fock/DFT functionals has been demonstrated to be crucial for a reliable calculation of the wavenumbers of vibrational modes in crystals. (Zicovich-Wilson et al. 2004; Demichelis et al. 2009; Ungureanu et al. 2010, 2012; De La Pierre et al. 2011; Prencipe et al. 2012; Prencipe 2014; Scanavino et al. 2012). The atomic basis sets employed are taken from the CRYSTAL online library, available at the web address www.crystal.unito.it/basis-sets.php. More precisely the 8-511G(d), 8-6311G(d) and 8-411G(d) contractions for $\mathrm{Mg}, \mathrm{Si}$ and $\mathrm{O}$, are used respectively. Such basis sets are chosen for being successfully employed in previous calculations of structure and properties of silicates; in particular, the same basis sets were used in another work, cited above (Noel et al. 2006), for the ab initio calculation of vibrational properties of forsterite.

High temperature calculations are performed evaluating the vibrational frequencies at the unit cell at different volumes, as a consequence of an increasing range of temperature. Mid-infrared frequencies and intensities are calculated for volume estimated at $0 \mathrm{~K}, 300 \mathrm{~K}$ and $1000 \mathrm{~K}$ (extreme situations) taking into account the zero point energy.

The calculated equilibrium lattice parameters optimized at the WC1LYP level (static values: no zero point and thermal effects included; see below in the text) are reported in Table 1. Zero point and thermal pressures at $0 \mathrm{~K}, 300 \mathrm{~K}$ and $1000 \mathrm{~K}$ and their impact on the calculated structure of forsterite are estimated by calculating the full vibrational spectrum at the $\Gamma$ point at different unit cell volumes, according to the procedure described by Prencipe et al. (Prencipe et al. 2011). It consists of a standard statistical-thermodynamics approach where, within the limit of the quasiharmonic approximation, zero point and thermal pressures are evaluated and added to the static 
pressure at each cell volume $(\mathrm{V})$, to get the total pressure $(\mathrm{P})$ as a function of both $\mathrm{T}$ and $\mathrm{V}$. The resulting $\mathrm{P}(\mathrm{V}, \mathrm{T})$ curves are fitted by an appropriate Equation of state $(\mathrm{EoS})$, to get bulk modulus $\left(\mathrm{K}_{0}\right)$ and its derivative $\left(\mathrm{K}^{\prime}\right)$, at different temperatures. The employed equation of state $($ EoS $)$ is a third order Brich-Murnaghan equation. In Table 2 are listed the bulk modulus $\left(\mathrm{K}_{0}\right)$, its first derivative $\left(\mathrm{K}^{\prime}\right)$ and the volume $\left(\mathrm{V}_{0}\right)$ calculated at the static equilibrium and at the three different considered temperatures $0 \mathrm{~K}, 300 \mathrm{~K}$ and $1000 \mathrm{~K}$ including zero point effects. The calculations successfully reproduce the available experimental data especially at room temperature in a very good agreement. In Table 2 are also reported experimental data at $300 \mathrm{~K}$ according to Finkelstein et al. (2014), who measured $\mathrm{K}_{0}$ at 130.0 (9) $\mathrm{GPa}$, where we foresee $\mathrm{K}_{0}$ of $131.1 \mathrm{GPa}$.

Thermal expansion at $300 \mathrm{~K}$ is $2.43 \mathrm{~K}^{-1}$ with a $\partial \mathrm{K} / \partial \mathrm{T}$ of $0.0217 \mathrm{GPa} / \mathrm{K}$ according to a linear fit within the range of 300-800K. Moreover, according to the value found by Bouhifid et al. (1996), the calculated thermal expansion shows a good agreement at $400 \mathrm{~K}$, where we calculate $2.80 \mathrm{~K}^{-1}$ which is the same experimental value.

As regards the calculation of frequencies, we refer to a previous paper (Pascale et al. 2004) for a more explicit formulation of the method. Here we simply remind that, within the harmonic approximation, frequencies at the $\Gamma$ point are obtained by diagonalizing the mass weighted Hessian matrix, whose elements are the second derivatives of the full potential of the crystal with respect to mass weighted atomic displacements. The first derivatives of the energy with respect to the atomic positions are calculated analytically (Doll et al. 2001), whereas second derivatives are calculated numerically by setting to $0.003 \AA$ the nuclear displacements with respect to the equilibrium positions.

\section{ISOTOPES}

\section{Sample characterization}

Room and high temperature Raman spectra were taken from the San Carlo olivine. Few powdered grains of the studied sample were examined by X-ray diffraction (D2 Bruker diffractometer, operating at $30 \mathrm{kV}$ and $10 \mathrm{~mA}$, with a $\mathrm{CuK} \alpha$ radiation $\lambda=1.54178 \AA$ ). Intensity measurement were taken in steps of $0.02^{\circ}$ over a $2 \theta$ range from $10^{\circ}$ to $80^{\circ}$, with a counting time of $1 \mathrm{~s}$ per step.). No other phase than olivine was found. The cell parameters are determined by Rietveld analysis (Larson and Von Dreele 1994; Toby 2001), were reported in Table 1. The determination of the unit cell parameters was performed by the Rietveld method with the GSAS-EXPGUI software package (Larson and Von Dreele, 2000; Toby, 2001). Independent variables for the Rietveld refinement are: zero point, nine coefficients of the shifted Chebyschev function to fit the background, unit cell dimensions and scale factor. A pseudo-Voigt profile function was used, refining one Gaussian $(\mathrm{Gw})$ 
and two Lorentzian (Lx and Ly) coefficients. The Rietveld analysis is done using structural data Hazen (1976. A good fit of the observed vs calculated patterns is obtained, as $\chi^{2}=1.5$.

Wavelength-dispersive spectrometer (WDS) microprobe analysis was done on few sample chips embedded in epoxy. The analyses are carried out using a CAMECA CAMEBAX SX50 electron microprobe, with a fine-focused beam ( $\sim 1 \mu \mathrm{m}$ diameter), an acceleration voltage of $15 \mathrm{kV}$ and a beam current of $20 \mathrm{nA}$, with $10 \mathrm{~s}$ counting times for both peak and total background, following the experimental procedures reported in Ferrari et al. (2014). Ten analytical spots are taken and merged. The resulting formula unit is $\mathrm{Mg}_{1.76} \mathrm{Fe}_{0.22} \mathrm{SiO}_{4}$, with compositional dispersion below 0.01 a.p.f.u. $\mathrm{Ca}, \mathrm{Cr}$ and $\mathrm{Al}$ concentration is below 0.002 a.p.f.u.

\section{Raman measurements}

The Raman measurements were performed at Institute of Planetary Research, DLR, Berlin, with a confocal Raman microscope Witec alpha300R system. The laser excitation wavelength was $532 \mathrm{~nm}$ and the spectral resolution about $4 \mathrm{~cm}^{-1}$ (600 lines/mm grating). A Nikon 10x objective is used and the spot size on the sample in focus was of about $1.5 \mu \mathrm{m}$. A laser power of $7 \mathrm{~mW}$ was chosen. The spectrum is the result of a time series of 50 single spectra with $10 \mathrm{~s}$ acquisition time for each single spectrum. For each measurement series the Rayleigh line of the scattered laser emission was adjusted to a zero position on the relative wavenumber unit scale. Furthermore, an edge filter was used to reduce the Rayleigh line. For data interpretation a Gaussian fit was applied to find the exact position of the maximum for each peak.

\section{Results and discussion}

The primitive unit cell of forsterite, $\mathrm{Mg}_{2} \mathrm{SiO}_{4}$, contains 28 atoms, giving rise to 84 normal modes, three of which correspond to pure translations. Under the Pbnm space group (n. 62) of the crystal, the vibrational representation $\Gamma_{\text {tot }}$ at the centre of the Brillouin zone may be reduced as follows:

$$
\Gamma_{\text {tot }}=11 A_{g}+11 B_{1 g}+7 B_{2 g}+7 B_{3 g}+10 A_{u}+10 B_{1 u}+14 B_{2 u}+14 B_{3 u}
$$

where the three translations are not taken into account. The 36 Raman active modes have either $\mathrm{A}_{\mathrm{g}}$ or $\mathrm{B}_{1 \mathrm{~g}}, \mathrm{~B}_{2 \mathrm{~g}}$ and $\mathrm{B}_{3 \mathrm{~g}}$ symmetry. All the calculated frequencies with their relative symmetry are listed in table 3, where are also reported frequencies and symmetry of volume cells calculated at $0 \mathrm{~K}$, $300 \mathrm{~K}$ and $1000 \mathrm{~K}$ including zero point effects (for calculations at high temperature see further in the discussion paragraph about Raman shifts due to temperature). 
Forsterite has been extensively investigated by mean of Raman spectroscopy, nonetheless, uncertainty about symmetry and mode assignments remain an open issue [...]. Data from literature are listed in table 4. Here are reported three data sets of wavenumbers taken from previous Raman studies taken on synthetic forsterite from Chopelas [..], Gillet et al. [..] and Kolesov and Geiger [..], in the fourth column are reported wavenumbers from the experimental measurements on the sample studied. Next to each column is reported the $\Delta v\left(\mathrm{~cm}^{-1}\right)$ resulting from the comparison with the calculated wavenumbers for forsterite, listed in the fifth column. The last five columns report the calculated isotopic shifts for $\mathrm{Mg}$ in M2 site, $\mathrm{Si}$ and the three O.

The assignment was done by comparing intensities and peak positions, which improves previous assignments based only on the calculated wavenumber, especially for modes at low wavenumbers. In figure 1, the experimental spectrum of forsterite $\left(\mathrm{FO}_{\# 89}\right)$ powder sample is compared with the calculated endmember. The box within the figure emphasizes wavenumbers within 150 to $500 \mathrm{~cm}^{-1}$, where a correct comparison can be done with calculations. To note, the spectra modelled is a perfect virtually infinite crystal, and the simulation of an unpolarised spectrum of a powder sample of forsterite will not take into account preferential orientations or grain size effects which could occur in an experimental measure. Peak at $304 \mathrm{~cm}^{-1}$ is an example. In measurements, it occurs with higher intensities compared to calculations. One reason could be crystal powder orientation.

For what concerns literature data, the maximum discrepancies on absolute average is around $7 \mathrm{~cm}^{-1}$, which is within the range of experimental error for each set of experimental data considered. The maximum discrepancy is around $-33 \mathrm{~cm}^{-1}$, for the mode at $378 \mathrm{~cm}^{-1}$. However, it is possible to claim a correct assignment of the mode, since the enlarged box in figure 1 shows a correspondence between the two peaks, taking into accounts intensity.

Moreover, it is possible to notice that the general discrepancies are spread for each set at the same wavenumbers, for example: the first wavenumber generally found in literature, and also in this study, is around $183 \mathrm{~cm}^{-1}$. In table 3, where are listed all the 36 foreseen Raman wavenumbers, wavenumbers around $183 \mathrm{~cm}^{-1}$ are calculated, but they have a different symmetry. In literature is consolidated that the first wavenumber found in forsterite has an $\mathrm{A}_{\mathrm{g}}$ symmetry, therefore the correct comparison with calculated data will be with the first $A_{g}$ mode, which is foresees at $191 \mathrm{~cm}^{-1}$. Another example is certainly the diagnostic peak that is frequently found experimentally around $824 \mathrm{~cm}^{-1}$, but which is calculated at $819 \mathrm{~cm}^{-1}$. 
Isotopic shift and mode assignment

The spectra after isotopic substitution of the six symmetry-independent atoms in the unit cell were calculated after substitution of ${ }^{56} \mathrm{Fe}$ for ${ }^{24} \mathrm{Mg}$ within the M2 and M1 sites, ${ }^{30} \mathrm{Si}$ for ${ }^{28} \mathrm{Si}$, and three ${ }^{18} \mathrm{O}$ for the three ${ }^{16} \mathrm{O}$ within the tetrahedron. As remarked into the computational details paragraph, the mass substitution is not a recalculation of the geometry of the system, but it only takes into account a same structure with an enlarged mass elements. The results, expressed as Raman $\operatorname{shift}\left(\mathrm{cm}^{-1}\right)$, are represented numerically in table $\mathbf{4}$ and graphically in figure $\mathbf{2 a}$ and $\mathbf{2 b}$ (the spectra has been divided into two parts to emphasize the peaks at low wavenumbers). Doing so, it can be found in the larger frequency shift, the larger the participation of the substituted atom to the movement; viceversa, zero frequency shift indicates that the substituted atom is not participating to the mode. It must be noticed that in both table and the figures $\mathrm{Mg} 1$ is not taken into accounts. Cations in M1 site are not Raman active. According to selection rules for the corresponding space group of forsterite, Pnma (n.62), listed in table 5, Mg1, which has 4a position in Wycoff symbol, does not have any Raman modes active for this space group.

With this tool is possible to establish the nature of each atomic motion in a given mode. For example, looking at table 4 and in particular the $\mathrm{Mg} 2$ column, forsterite spectra can be divided quantitatively in three possible regions, confirming what is extensively remarked in literature (for clarity, from now on we are going to refer to calculated wavenumbers). A first one that goes from $191 \mathrm{~cm}^{-1}$ to $351 \mathrm{~cm}^{-1}$. Here the $\mathrm{Mg}$ within the M2 site, vibrates mainly, with small contributions of oxygens and a negligible contribution of silicon, confirming what in literature is defined as a region of rigid rotation and translation of the lattice, due to $\mathrm{SiO}_{4}$ and $\mathrm{M} 2$ translation. The highest motion of $\mathrm{Mg} 2$ is calculated at $351 \mathrm{~cm}-1$. Other three high Raman shifts are calculated respectively at $228 \mathrm{~cm}-$ 1, $304 \mathrm{~cm}-1$ and $324 \mathrm{~cm}-1$. These data overlap with Kolesov and Geiger that, in their experimental isotopes substitution of ${ }^{26} \mathrm{Mg}$ for ${ }^{24} \mathrm{Mg}$, found the biggest shifts due to $\mathrm{Mg}$ at peak at $304 \mathrm{~cm}^{-1}$ and $329 \mathrm{~cm}^{-1}$.

The second region

The third region

Si-O stretching modes and $\mathrm{Si}-\mathrm{O}$ bond distances

Peaks at high wavenumbers, which are less sensitive to temperature and pressure can be linked to the crystalline structure studying Raman shifts [...]. In figure 3 are shown bond distances of Si-O1, $\mathrm{Si}-\mathrm{O} 2$ and $\mathrm{Si}-\mathrm{O} 3$, taken from Kudoh et al., measured at increasing pressure (here considered from 
1bar to $80 \mathrm{kbar}$ ), plotted against Raman shifts calculated from Chopelas at those same pressures. In the figures are shown the trends of just three peaks at high wavenumbers, which are diagnostic for forsterite and in this work are calculated at ambient conditions at $819 \mathrm{~cm}-1,857 \mathrm{~cm}-1$ and $920 \mathrm{~cm}-1$ (in figure plotted as stars). The linear fits of the three trends show slightly different slopes for the first two linear fits at $819 \mathrm{~cm}-1$ and $857 \mathrm{~cm}-1$ and a much more tilted slope for the peak at $920 \mathrm{~cm}-1$. The reason may be found in bond distances between $\mathrm{Si}$ and $\mathrm{O}$ within the tetrahedral for forsterite. At ambient conditions we have from Kudoh et al. that the distances are respectively for Si-O2 1.654 $\AA$, for Si-O3 $1.635 \AA$ and for Si-O1 $1.614 \AA$. As we can see from figure $3 \mathbf{b}$ those three peaks are characterized by the highest contributions in the motion of oxygens which are respectively $\mathrm{O} 2$ for $819 \mathrm{~cm}-1$, O3 for $857 \mathrm{~cm}-1$ and $\mathrm{O} 1$ for $920 \mathrm{~cm}-1$. Therefore what can be assessed is that for shorter bond lengths correspond higher wavelengths and thus an higher energy, if compared to longer bond distances which are set at shorter wavelengths and thus lower energy.

\section{Calculated Raman shift due to temperature}

In figure 4 are presented in offset the three spectra calculated respectively at $0 \mathrm{~K}, 300 \mathrm{~K}$ and $1000 \mathrm{~K}$. The details of calculations are reported in the previous computational details paragraph, in which is remarked the accuracy of calculations, taking into accounts the calculated thermal expansions if compared with experimental data in literature [..], or if compared with $\mathrm{K} 0, \mathrm{~K}$ ' and volume of the unit cell with one of the latest study done on forsterite [..] (see table 2).

We compared the slope $\Delta v \mathrm{j} / \Delta \mathrm{T}$ calculated from the difference peak positions at 0 and $1000 \mathrm{~K}$ of our peaks and the experimental $\delta v \mathrm{j} / \delta \mathrm{T}$ obtained by Gillet et al. up to $1150 \mathrm{~K}$ for the strongest and best measured peaks at $819,857,920$ and $974 \mathrm{~cm}-1$. The agreement is within experimental error, suggesting that calculated frequencies at higher temperature can be used to predict the atual changes in Raman peak positions.

\section{Conclusions}

The simulation of the Raman spectrum provided by combined DFT with a Hartree-Fock exchange contribution is very close to the experimental spectrum, both in peak position and intensities. It shows that quantum mechanical modelling can be used to interpret the observed experimental spectra and find links between crystal structure and spectroscopic feature. Going further, the simulation provides a theoretical framework in which is possible to model the observed spectra, especially in those where a correct assignment is hinder by low intensities and mixed modes a low wavenumbers, in cases such as forsterite. 
We have shown that quantum mechanical calculations, combined with a careful description of the modes and exploiting some zero computational cost tools of CRYSTAL14 (i.e. isotopic), enable to assess the influence of bond distances and cation masses on a given mode. Therefore, we can assume which modes are pressure, temperature or composition sensitive, whose wavenumbers are related to the structural changes with pressure, temperature and composition. The good agreement between the calculated and the experimental data allow us to formulate the following conclusions that the calculated data foresee a similar trend if compared to experimental measurements: according to the increasing temperature (from $0 \mathrm{~K}$ to $1000 \mathrm{~K}$ ) the wavenumbers decrease. It means that the temperature factor must be taken into account and the calculations are accurate.

We believe that our approach of combining experimental structural data and $a b$ initio quantum mechanical calculation, already employed also in other minerals and mineral families, could be a powerful tool to create a consistent physical background for Raman data interpretation, not only for mode assignment, but also to foresee temperature behaviour, thus useful to develop methods within the field of planetary science. 